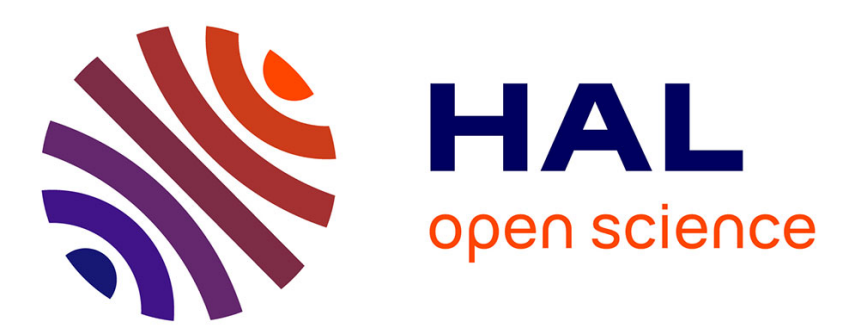

\title{
Novel signature of chaos in quantum-mechanical states
}

\author{
F. Leyvraz, J. Quezada, T. H. Seligman, M. Lombardi
}

\section{To cite this version:}

F. Leyvraz, J. Quezada, T. H. Seligman, M. Lombardi. Novel signature of chaos in quantum-mechanical states. Physical Review Letters, 1991, 67, pp.2921-2925. 10.1103/PHYSREVLETT.67.2921 . hal-00974253

\section{HAL Id: hal-00974253 \\ https://hal.science/hal-00974253}

Submitted on 5 Apr 2014

HAL is a multi-disciplinary open access archive for the deposit and dissemination of scientific research documents, whether they are published or not. The documents may come from teaching and research institutions in France or abroad, or from public or private research centers.
L'archive ouverte pluridisciplinaire HAL, est destinée au dépôt et à la diffusion de documents scientifiques de niveau recherche, publiés ou non, émanant des établissements d'enseignement et de recherche français ou étrangers, des laboratoires publics ou privés. 


\title{
PHYSICAL REVIEW \\ LETTERS
}

\begin{tabular}{lcc}
\hline Volume 67 & 18 NOVEMBER 1991 & NuMBER 21 \\
\hline
\end{tabular}

\section{Novel Signature of Chaos in Quantum-Mechanical States}

\author{
F. Leyvraz, J. Quezada, and T. H. Seligman \\ Instituto de Física, Laboratorio de Cuernavaca, University of Mexico (UNAM), Apdo. postal 20-364, \\ 01000 Mexico Distrito Federal, Mexico \\ M. Lombardi \\ Laboratoire de Spectrométrie Physique, Université Joseph Fourier-Grenoble 1, B.P. 87, \\ 38402 Saint-Martin-d'Hères CEDEX, France \\ (Received 24 May 1991)
}

\begin{abstract}
A quantity related to the elastic enhancement in nuclear reactions is introduced to analyze how the transition from integrable to chaotic behavior in a classical system is reflected in the eigenstates of the corresponding quantum system. Its behavior is first studied for random matrix models believed to represent such a transition, namely, a band matrix ensemble as well as a Poisson ensemble perturbed by a Gaussian orthogonal ensemble. Both of these display similar behavior. We then show that the same characteristics may be found in a more realistic system showing such a transition, namely, molecular Rydberg states calculated for high angular momenta.
\end{abstract}

PACS numbers: 05.45.+b, 03.65.Ge, 05.40. $+\mathrm{j}$

In recent years there has been considerable interest in the behavior of quantum systems, the classical counterpart of which show chaotic behavior. Most of these studies focused on the energy spectrum [1-3]. The Gaussian orthogonal ensemble (GOE) has proven to be a good model for the fluctuation properties of such spectra. The spectra of Hamiltonians with a behavior intermediate between integrable and chaotic have also been studied by similar models [4] with good agreement. On the other hand, the situation is far more confusing with respect to the eigenstates of such Hamiltonians. The reason for the difficulty is the arbitrariness in the choice of basis. In particular, many peculiar and system-dependent features, such as "scars," have been observed in specific bases (coordinate space [5], Wigner functions [6], and others). However, a general characteristic of states displaying the universal aspects of the transition from order to chaos is still absent.

To this end we consider two arbitrary orthogonal vectors $\phi$ and $\psi$, and construct the following quantity:

$$
q(t)=\frac{|\langle\psi \mid \psi(t)\rangle|^{2}}{|\langle\phi \mid \psi(t)\rangle|^{2}},
$$

where $|\psi(t)\rangle$ denotes $e^{i H t}|\psi\rangle$ and $H$ is the Hamiltonian under consideration. The quantity we are interested in will be the time average $Q$ of $q(t)$ for large times. The physical interpretation of this quantity is straightforward: it denotes the degree to which an arbitrary state will overlap with itself at large times, compared to the degree with which it will overlap with any other state orthogonal to it. This is related to the well-known concept of elastic enhancement in the weak absorption limit of nuclear reactions. An appropriate energy average of this quantity is usually expected to be equal to 3 [7], as will be confirmed by our findings.

If we denote by $\phi_{i}$ and $\psi_{i}$ the components of $\phi$ and $\psi$ with respect to the eigenbasis of $H$ and $N$ is the dimension of the underlying Hilbert space, one finds

$$
Q=\frac{\sum_{i=1}^{N}\left|\psi_{i}\right|^{4}}{\sum_{i=1}^{N}\left|\psi_{i}\right|^{2}\left|\phi_{i}\right|^{2}} \text {. }
$$

This only depends on the orientation of $\phi$ and $\psi$ with respect to the eigenbasis, and not at all on the behavior of the eigenvalues. If the quantity $Q$ is averaged over all vectors, the value obtained is very close to 3 , for large $N$, independent of the properties of $\boldsymbol{H}$. If the denominator and the numerator are averaged over separately before taking the quotient, it has also been shown [8] that $Q$ is exactly 3 for any $N$.

Yet beyond these properties we find a self-averaging 
behavior which is due to the sum in Eq. (2). Indeed in the limit of $N \rightarrow \infty$ the value of $Q$ is 3 for almost any pair of vectors on the unit sphere. This follows from two considerations [7], both valid in the limit of large $N$ : First, the coordinates of any vector are in a first approximation independently Gaussian distributed; second, the coordinates of two orthogonal vectors are also independent of each other. Both of these remarks become intuitively clear, if it is noted that the only constraints on the coordinates are normalization and orthogonality. Thus one finds, up to higher-order terms in $N$, that $\sum_{i=1}^{N}\left|\psi_{i}\right|^{4}$ is equal to $N\left\langle\left\langle\left|\psi_{1}\right|^{4}\right\rangle\right\rangle$ and $\sum_{i=1}^{N}\left|\psi_{i}\right|^{2}\left|\phi_{i}\right|^{2}$ to $N\left\langle\left\langle\left|\psi_{1}\right|^{2}\left|\phi_{1}\right|^{2}\right\rangle\right\rangle$, where the double angular brackets denote an average over a Gaussian distribution. This proves our statement and incidentally also the much weaker statement made in the previous paragraph.

From this follows that $Q$ gives an indication as to whether a pair of vectors is random with respect to the eigenvectors of $\boldsymbol{H}$ or not. In the former case, the value of $Q$ should be 3 whereas in the second case it should be different and generally large [9]. Thus the following test for chaos can be performed: Consider two orthogonal wave packets localized with respect to some particular observable (e.g., position, momentum, or any combination of these). The value of $Q$ for these two wave packets should be 3 in a chaotic system, since there is no relation between the eigenfunctions of $H$ and any wave packet defined independently from the eigenfunctions of $H$. On the other hand, in an integrable system, the value may be much larger, since semiclassical wave functions correspond roughly to wave packets localized on the tori of the classical system. In this sense, the quantity $Q$ is similar to the inverse participation ratio, which measures the localization in a given basis. Indeed, the numerator of $Q$ is exactly the inverse participation ratio. Note that in this particular way of using $Q$, we have not entirely gotten rid of the basis dependence, since we must choose the wave packets according to some criterion usually dictated by the physics of the system. We shall return to this question later.

Up until now we have discussed the case of a specific Hamiltonian. Let us consider, instead, what happens if we look at ensembles of Hamiltonians such as the GOE and its variants that have been used to describe transition from order to chaos. The GOE is defined as the ensemble of matrices having all off-diagonal elements independently Gaussian distributed with the same variance and mean zero and the diagonal elements are distributed independently with twice the variance. This gives rise to an ensemble of matrices, the eigenvectors of which are uniformly distributed over the unit sphere.

To study the transition from order to chaos the following models have been used: To describe an integrable system we use an ensemble of matrices with fixed eigenvectors and random independent eigenvalues. To describe intermediate situations, one uses the two following models: the Porter-Rosenzweig model [10], where one takes

$$
h_{i j}=g_{i j}\left[\delta_{i j}+\lambda\left(1-\delta_{i j}\right)\right],
$$

where $g_{i j}$ denotes matrix elements from a GOE matrix and $\lambda$ is a transition parameter. We also consider the band matrix model [4], which is given by

$$
h_{i j}=g_{i j} \exp \left[-(i-j)^{2} / \sigma^{2}\right] \text {, }
$$

where $\sigma$ now plays the role of the transition parameter. Note that neither of these definitions is basis invariant. In particular, in both of these ensembles, the eigenvectors are in some sense localized with respect to the particular basis in which they are defined. The way in which this takes place, however, is quite different in the two cases: In the band matrix model, the eigenvectors have a peak at one given point $i$ and decay as the distance to $i$ increases. In the Porter-Rosenzweig model, on the other hand, the eigenvectors consist of one peak at a point $i$ together with a weak background uniformly distributed over all other basis vectors. In this case, it is not obvious how to define such a concept as that of localization length, for example.

In studying ensembles, as opposed to isolated Hamiltonians, there is a basis-independent characterization of chaos, as we now show. To see this, fix two orthogonal vectors $|\phi\rangle$ and $|\psi\rangle$ and then proceed to evaluate the distribution of $Q$ given the distribution of $H$. If the ensemble of $H$ is a GOE, the distribution of $Q$ is going to be the same as the distribution of $Q$ for an ensemble of vectors uniformly distributed over the unit sphere. That is, it is going to be fairly sharply peaked at a value of 3 , and will have a variance that goes to zero as const $\times N^{-1 / 2}$. Yet this variance cannot at all be neglected in practical situations. Figure 1 shows that the variance is still significant for $N=160$, and thus the constant is quite large.

In cases where the ensemble is intermediate, however, some distinctive features appear. First, the distribution of $Q$ is no longer independent of the vector pair chosen. Rather, it shows an increasing degree of variability as the integrable case is approached. In Fig. 2 we display the

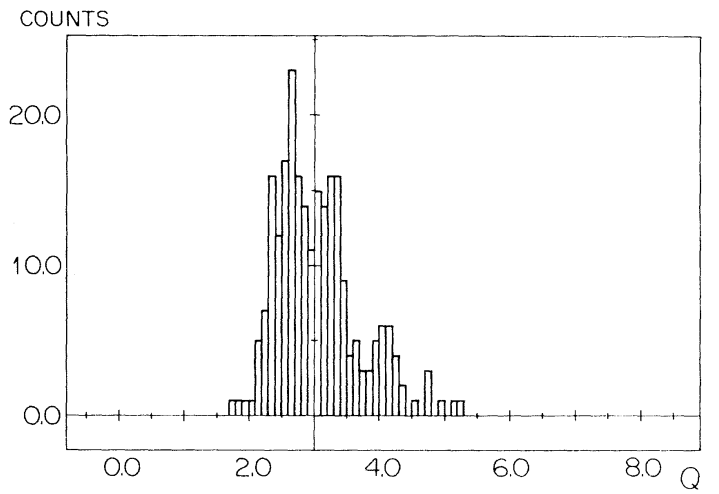

FIG. 1. Distribution of $Q$ for a GOE with the space dimension equal to 160 . 


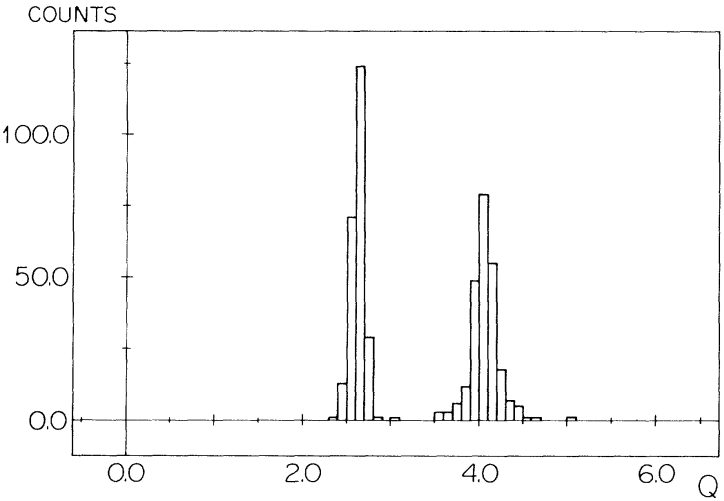

FIG. 2. Distribution of $Q$ for two vector pairs in the band matrix model with $\sigma$ equal to 0.5 and space dimension equal to 160. Note the variation in the shape, as well as the difference from Fig. 1.

distribution of $Q$ for an intermediate case. We note the following features: First, the distribution of $Q$ changes quite markedly as we vary the vector pair.

To be more quantitative we now consider the average $M(\phi, \psi)=\langle Q\rangle$ and the variance $\Sigma(\phi, \psi)=\left\langle Q^{2}\right\rangle-\langle Q\rangle^{2}$, where \langle\rangle indicates an average over the ensemble of Hamiltonians. These two quantities, considered for different vector pairs $\phi, \psi$, have a significant correlation as may be seen in Table I. Furthermore, the variance has a tendency to increase as we go from integrable to chaotic. Indeed for a fixed pair of vectors, $Q$ shows no variance at all in the Poisson ensemble. This follows from the fact that the eigenbasis in the Poisson ensemble is taken to be fixed and that $Q$ only depends on the coordinates of the two vectors in the eigenbasis [see Eq. (2)]. As the system becomes more and more chaotic, the eigenbasis varies more and more, and so does $Q$. However, it should be said that this tendency is not always unambiguous. In the intermediate regime, $\Sigma(\phi, \psi)$ can be quite large. It also then has an anomalously large average value, and the distribution function will be markedly different from the one observed for the GOE.

To complete our quantitative understanding we can

TABLE I. Mean and variance of the distribution of $Q$ for various values of $N$ and $\sigma$ for the band model. We have also performed the same study on the Porter-Rosenzweig model, with virtually identical results.

\begin{tabular}{cccc}
\hline \multicolumn{2}{c}{$(N=80, \sigma=2)$} & \multicolumn{2}{c}{$(N=40, \sigma=1)$} \\
$M(\psi, \phi)$ & $\Sigma(\psi, \phi)$ & $M(\psi, \phi)$ & $\Sigma(\psi, \phi)$ \\
\hline 2.466 & 0.183 & 2.187 & 0.172 \\
2.561 & 0.210 & 2.692 & 0.455 \\
3.005 & 0.273 & 3.175 & 0.756 \\
3.620 & 0.440 & 3.758 & 0.609 \\
3.743 & 0.57 & 4.415 & 1.6603 \\
4.154 & 0.581 & 5.027 & 1.510 \\
\hline \hline
\end{tabular}

next study the behavior of $M$ and $\Sigma$ as we average over the vector pairs $\phi, \psi$. Such averages will be indicated by overlining. Consider the quantities $\bar{M}, \operatorname{var}(M)=\bar{M}^{2}$ $-\bar{M}^{2}, \bar{\Sigma}$, and $\operatorname{var}(\Sigma)=\bar{\Sigma}^{2}-\bar{\Sigma}^{2}$. For a GOE $\operatorname{var}(M)$ is zero and becomes increasingly larger as we approach integrability. The size of this signature decreases with $N^{-1}$, in consequence of the fact that $Q$ is almost always equal to 3 for large $N$, independently of the ensemble. Close to integrability, however, the tail of the distribution is noticeable (with good statistics) even for fairly large $N$. Table II shows these four quantities for $N=160$ for different values of the transition parameter $\sigma$ in the band model, together with similar quantities for a physical system discussed below.

As we may expect from the above remarks, $\bar{M}$ is always about equal to 3 and $\operatorname{var} M$ decreases as one goes from integrable to chaotic. $\bar{\Sigma}$ is very small for a nearintegrable situation and increases monotonically to its GOE value, but $\operatorname{var}(\Sigma)$ has a maximum value somewhere between complete chaos and integrability. It must be zero for both extremes.

Thus we have found quite well-defined signatures of chaos for ensembles of Hamiltonians. Yet if we wish to apply this to a fixed Hamiltonian, we will have to invoke ergodicity in some way to replace the ensemble average by an energy average as we usually do when discussing spectra. But here we find a difficulty: How do we maintain this "fixed pair of vectors" which we use throughout our previous argument when varying the energy? Let us first turn to our starting point, the elastic enhancement in nuclei. There the functions in question are channel functions defined at some distance from the interaction region-we could imagine in a way a projection of the internal functions on a channel hypersphere. The radial function which determines the energy is integrated over in each channel and thus the overlap with any given channel is well defined for all energies far from thresh-

TABLE II. The four characteristic quantities $\bar{M}, \operatorname{var}(M), \bar{\Sigma}$, and $\operatorname{var}(\Sigma)$, given for $N=160$ and various values of $\sigma$ for the band model as well as for Rydberg molecules in the "nearintegrable" and chaotic cases. Note that $\bar{\Sigma}$ is much larger than for the band matrix model because there $N$ is 41 rather than 160 . In both cases, in the chaotic regime, $\operatorname{var}(\Sigma) / \bar{\Sigma}$ does not become zero because of the finite number of vector pairs used for the average (160 and 10, respectively).

\begin{tabular}{ccclc}
\hline \hline$\sigma$ & $\bar{M}$ & $\operatorname{var}(M)$ & \multicolumn{1}{c}{$\bar{\Sigma}$} & $\operatorname{var}(\Sigma)$ \\
\hline 0.5 & 3.31 & 0.526 & 0.017 & 0.009 \\
1.0 & 3.39 & 0.52 & 0.21 & 0.078 \\
2.0 & 3.163 & 0.423 & 0.316 & 0.120 \\
8.0 & 3.097 & 0.091 & 0.39 & 0.046 \\
$\infty$ & 3.068 & 0.014 & 0.365 & 0.030 \\
Rydberg molecules & & & & \\
Integrable case & 3.17 & 0.69 & 1.07 & 0.68 \\
Chaotic case & 3.22 & 0.24 & 1.5 & 0.95 \\
\hline \hline
\end{tabular}


olds.

Applying this idea, say, to a billiard we could think of wave packets with fixed location and a wave vector $\mathbf{k}$ with given angle but variable absolute value $k$. $k$ will determine the energy of this state, and, invoking ergodicity, we can simulate an average over an ensemble of Hamiltonians by averaging over $k$, for such a wave packet or any superposition thereof. Averages over vector pairs, as discussed above, would have to be averages over pairs of random superpositions of such packets.

We shall try to implement such a program for Rydberg molecules. A Rydberg molecule is considered as a cylindrically symmetric charge with a net value of +1 as a core and a single electron moving on Rydberg-type orbits whenever it is far from the core. For periods of the electron orbit of the same order of magnitude as those of the core the Born-Oppenheimer approximation breaks down. The absolute value $L$ of the electronic angular momentum $\mathbf{L}$ is approximately conserved but the rotational motion of the core and the radial motion of the electron are coupled and thus one (approximately) conserved quantity is missing to make the system (almost) integrable and thus we can find chaos for 2 degrees of freedom. The classical limit of the approximate system that conserves $L$ exactly can be constructed [11] and its behavior discussed. If the frequencies of the core and electron are in resonance, the system becomes near integrable, as the Born-Oppenheimer approximation is again almost valid due to the stroboscopic effect [11]. In this case Kolmogorov-Arnol'd-Moser (KAM) tori are only destroyed in a very small region. Off resonance and choosing an adequate coupling constant we find essentially complete classical chaos.

This simplified classical model was derived as a limit from the multichannel quantum defect method (MQDT) which allows fairly simple calculations of eigenvalues and of the projection of eigenfunctions on the $(2 L+1)$ dimensional space spanned by the angular part of the electron wave function. This space splits due to invariance of the potential with respect to reflection by a plane containing the molecular axis, into a $(L+1)$ - and $L$ dimensional subspace [11]. Taking this into account one conveniently introduces a labeling of the basis in terms of projections $\Lambda$ of $L$ on the molecular axis. While experiment at this point corresponds to fairly small values of $L$, we chose the $(L+1)$-dimensional subspace for $L=40$ in order to have a larger number of projections. The calculations are discussed in detail in Ref. [12]. A quasiperiodicity of $L+1$, corresponding to a unit increase of the average principal quantum number $n$ of the Rydberg series, dominates spectra and intensities in both the regular and irregular domains of classical motion.

To test our methods on this system, we have performed the following calculations: First, we considered states with fixed value of $\Lambda$. For these it was found that the value of $Q$ was, to a good approximation, equal to 3 in the classically chaotic regions, whereas it rose to exceedingly high values $(Q \gg 1000)$ in the "near-integrable" regions. This indicates that the eigenstates of the Hamiltonian can truly be thought of as random in the chaotic region, at least in the angular momentum basis, whereas this is not the case in the integrable region and thus the basisdependent test proposed at the beginning of this paper works very well in this case.

We next proceed to apply the basis-invariant tests proposed for ensembles following the ideas put forward above. We divide the spectrum into blocks of size 41 , and replace the average over ensembles of Hamiltonians — invoking ergodicity - by averages over blocks. Because of the "near periodicity" in spectral properties mentioned above, it is reasonable to assume that two vectors in different blocks, but with identical projections, are indeed "the same" with respect to this particular Hamiltonian.

We can then proceed to calculate the quantities $\bar{M}$, $\operatorname{var}(M), \bar{\Sigma}$, and $\operatorname{var}(\Sigma)$. The corresponding results are shown at the end of Table II both for the very chaotic off-resonance and for the near-integrable on-resonance case. The average over pairs, indicated by the overbar, is taken within each of these forty-one-dimensional subspaces defined by the blocks.

Comparing the results for Rydberg molecules in Table II with those for the random matrix ensembles we find the expected behavior for all quantities if we take into account the poor statistics. The results for molecules are only shown for the two extreme cases, because the transition from order to disorder is rather abrupt in this case. The present numerical results are insufficient to determine the quantities in question for the transition, although more extensive calculations for Rydberg molecules near resonances are planned.

For a specific physical system the question of the existence of scars [5] arises. The question how the criteria developed here behave with respect to scars can only be answered by studying a system whose scar structure is known. This structure has not yet been well established for Rydberg molecules, although the near periodicity is a hint that some unusual structures must exist. These will be analyzed to some extent in Ref. [12], but at this point we can only say that the numbers we find in the last two rows in Table II are consistent with insensitivity to, or absence of, scars.

Summarizing, we have found criteria for the transition from a GOE to a Poisson ensemble of Hamiltonians that can be applied in a basis-independent fashion to wave functions. Furthermore, we have pointed out sufficient criteria for single Hamiltonians. To apply them either one has to infer something about KAM tori and thus introduce a weak basis dependence or one has to be able to perform an energy average by formulating a family of states or projectors that can reasonably be "shifted" in energy, i.e., a way must be found to transform a given vector or projection to a different value of the energy without changing its essential characteristics. While this is clearly somewhat vague, in practical applications such 
a way can usually be found if the system scales over a sufficiently broad range of energies, which is a necessary condition even for the analysis of spectra. For Rydberg molecules it was shown that both types of criteria can be successfully applied.

The authors gratefully acknowledge valuable conversations with P. Mello as well as financial assistance from CIFMA A.C.

[1] O. Bohigas, M. J. Giannoni, and C. Schmit, Phys. Rev. Lett. 52, 1 (1984).

[2] T. H. Seligman and J. J. M. Verbaarschot, Phys. Lett. 108A, 183 (1985).

[3] M. V. Berry and M. Robnik, J. Phys. A 17, 2413 (1984).

[4] T. H. Seligman, J. J. M. Verbaarschot, and M. R. Zirnbauer, Phys. Rev. Lett. 53, 215 (1984); J. Phys. A 18,
2751 (1986).

[5] E. Heller, Phys. Rev. Lett. 53, 1515 (1984); in Proceedings of the International Conference of Cuernavaca, edited by T. H. Seligman and H. Nishioka, Lecture Notes in Physics Vol. 263 (Springer-Verlag, Berlin, 1986); E. G. Bogolmolny, Physica (Amsterdam) 31D, 169 (1988).

[6] M. V. Berry, Proc. R. Soc. London A 423, 219 (1989).

[7] T. A. Brody, J. Flores, J. B. French, P. A. Mello, A. Pandey, and S. S. M. Wong, Rev. Mod. Phys. 53, 385 (1981).

[8] N. Ullah and C. E. Porter, Phys. Lett. 6, 30 (1963).

[9] If both vectors are strongly localized in the same area, it is possible to find smaller values. This is quite unlikely in practice.

[10] N. Porter and C. E. Rosenzweig, Phys. Rev. 120, 1698 (1960).

[11] M. Lombardi, P. Labastie, M. C. Bordas, and M. Broyer, J. Chem. Phys. 89, 3479 (1988).

[12] M. Lombardi and T. H. Seligman (to be published). 